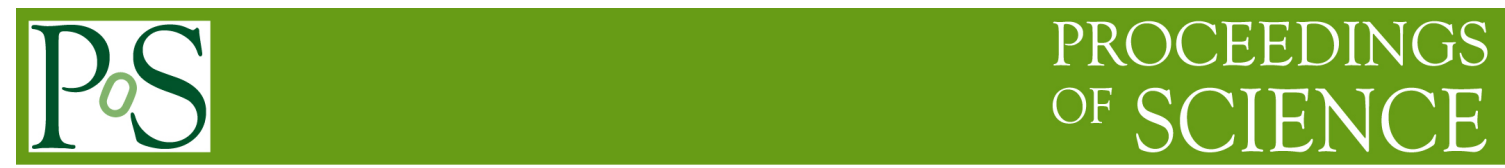

\title{
Complex Systems and Quantum Field Theory
}

\author{
Alexander Glück* \\ Faculty of Physics, University of Vienna \\ E-mail: alexander.glueck@chello.at \\ Helmuth Hüffel \\ Faculty of Physics, University of Vienna \\ E-mail: helmuth.hueffeleunivie.ac.at

\section{Saša Ilijić} \\ Department of Physics, Faculty of Electrical Engineering and Computing, University of Zagreb \\ E-mail: sasa.ilijicefer.hr
}

We present a recently established model in complex systems theory and give a formulation for infinite degrees of freedom. Our field theory is quantized via the Stochastic Quantization method. Applying this quantization procedure to Scalar QED, the Higgs potential arises in the equilibrium limit.

Black Holes in General Relativity and String Theory August 24-30 2008

Veli Lošinj, Croatia

\footnotetext{
*Speaker.
} 


\section{Brownian Motion and Quantum Field Theory}

Path integral quantization of field theories is analogous to the calculation of stochastic averages. Consider Brownian motion of a particle with coordinates $q^{i}$ in a given potential $U$, described via the Langevin equation [1]

$$
\frac{d q^{i}(t)}{d t}=-\delta^{i j} \frac{\partial U(q)}{\partial q^{j}}+\eta^{i}(t),
$$

where $\eta$ is a completey irregular function ("noise"), thus satisfying the correlations

$$
\left\langle\eta^{i}(t)\right\rangle=0 \quad, \quad\left\langle\eta^{i}(t) \eta^{j}(\bar{t})\right\rangle=2 \delta^{i j} \delta(t-\bar{t}) .
$$

Expectation values of a function of the coordinates can be calculated as follows:

$$
\langle f(q)\rangle \sim \int d^{n} q \rho(q, t) f(q) .
$$

The density $\rho$ is given by the Fokker Planck equation

$$
\frac{\partial \rho(q, t)}{\partial t}=\frac{\partial}{\partial q^{i}} \delta^{i j}\left(\frac{\partial U(q)}{\partial q^{j}}+\frac{\partial}{\partial q^{j}}\right) \rho(q, t),
$$

which has a simple solution for $\dot{\rho}=0$, the equilbrium distribution

$$
\rho_{\text {equ }}(q) \sim e^{-U(q)} .
$$

Equilibrium expectation values of a function $f(q)$ are therefore calculated to be

$$
\langle f(q)\rangle_{\text {equ }} \sim \int d^{n} q e^{-U(q)} f(q) .
$$

Now the crucial point is to compare the structure of this formula with the Green functions in Quantum Field Theory, which are expectation values of fields calculated in a similar way:

$$
G\left(x_{1}, \ldots, x_{n}\right) \sim \int \mathscr{D} \phi e^{-S[\phi]} \phi\left(x_{1}\right) \ldots \phi\left(x_{n}\right) .
$$

\section{Stochastic Quantization}

Green functions can thus be interpretated as equilibrium expectation values of a Brownian motion process, where the equilibrum distribution density is just the usual path integral density $e^{-S[\phi]}$. Indeed, a Langevin equation for a field $\phi=\left\{\phi^{i}\right\}$ can be formulated, such that its equilibrium limit gives the Green functions written above. It reads [2], [3], [4]

$$
\frac{\partial \phi(x, s)}{\partial s}=-\frac{\delta S}{\delta \phi}(x, s)+\eta(x, s)
$$

in analogy to equation (1.1). $s$ is the evolution parameter of the stochastic process, usually called "stochastic time". The noise field satisfies the relations

$$
\langle\eta(x, s)\rangle=0 \quad, \quad\langle\eta(x, s) \eta(\bar{x}, \bar{s})\rangle=2 \delta^{4}(x-\bar{x}) \delta(s-\bar{s}) .
$$


The Fokker Planck equation now reads

$$
\frac{\partial \rho[\phi, s]}{\partial s}=\int d^{4} x \frac{\delta}{\delta \phi}\left(\frac{\delta S[\phi]}{\delta \phi}+\frac{\delta}{\delta \phi}\right) \rho[\phi, s]
$$

whose equilibrium solution can be read of easily:

$$
\rho_{\text {equ }}[\phi] \sim e^{-S[\phi]} .
$$

Expectation values of a functional $\mathscr{F}[\phi]$ are therefore calculated in the following way:

$$
\langle\mathscr{F}[\phi]\rangle_{e q u} \sim \int \mathscr{D} \phi e^{-S[\phi]} \mathscr{F}[\phi] .
$$

Hence the Green functions of Quantum Field Theory are equilibrium averages for a field undergoing Brownian Motion as described by the field equation (2.1):

$$
G\left(x_{1}, \ldots, x_{n}\right) \sim \int \mathscr{D} \phi e^{-S[\phi]} \phi\left(x_{1}\right) \ldots \phi\left(x_{n}\right) .
$$

\section{Active Brownian Motion and Active Stochastic Quantization}

Equation (2.1) describes Brownian motion of a field $\phi$ in the potential $S[\phi]$. When the process equilibrates, this leads to a QFT with path integral density $e^{-S[\phi]}$. The action is typically $\sim \phi^{2}$, therefore defining linear dynamics of the field. One can ask the following question: What kind of QFT (i.e. what path integral density) will be the equilibrium of a certain nonlinear Brownian motion? A recently established, heavily studied model of nonlinear Brownian motion is "active" Brownian motion [5], [6], whose generalization for infinite degrees of freedom we studied in [7]. The Langevin equation for fields now reads:

$$
\frac{\partial \phi^{i}(x, s)}{\partial s}=-\delta^{i j} \frac{\delta S}{\delta \phi^{j}}(x, s)+e \delta^{i j} \frac{\partial V}{\partial \phi^{j}}(x, s)+\eta^{i}(x, s),
$$

where $e$ is an additional degree of freedom, called the "internal" energy, satisfying the equation

$$
\frac{\partial e}{\partial s}=c_{1}-c_{2} e-c_{3} e V(\phi)
$$

$c_{i}$ are parameters assumed to be positive. For a specific choice of these parameters, the internal energy, after some time, equilibrates to a fixed value: $\frac{\partial e}{\partial s}=0$ (as can be seen by a stability analysis of fixpoints or a direct computer simulation, see [8]), so that it becomes a pure function of the fields:

$$
e=e(\phi)=\frac{c_{1}}{c_{2}+c_{3} V(\phi)}
$$

Plugging this expression into the field equation (3.1) yields the standard form

$$
\frac{\partial \phi(x, s)}{\partial s}=-\frac{\delta \tilde{S}}{\delta \phi}(x, s)+\eta(x, s)
$$

with the effective action

$$
\tilde{S}[\phi] \equiv \int d^{4} x\left\{\mathscr{L}(\phi, \partial \phi)-\frac{c_{1}}{c_{3}} \ln \left(c_{2}+c_{3} V(\phi)\right)\right\} .
$$


The resulting Quantum Field Theory is given by the Green Functions

$$
G\left(x_{1}, \ldots, x_{n}\right) \sim \int \mathscr{D} \phi e^{-\tilde{S}[\phi]} \phi\left(x_{1}\right) \ldots \phi\left(x_{n}\right) .
$$

Active Brownian motion of fields in the equilibrium limit thus results in a QFT with the effective Lagrangian being given by the sum of the usual kinetic term $T(\partial \phi)$ and the effective potential $\tilde{V}(\phi)$ :

$$
\tilde{\mathscr{L}}(\phi, \partial \phi)=\mathscr{L}(\phi, \partial \phi)-\frac{c_{1}}{c_{3}} \ln \left(c_{2}+c_{3} V(\phi)\right)=T(\partial \phi)+\tilde{V}(\phi) .
$$

The effective potential $\tilde{V}(\phi)$ reads

$$
\tilde{V}(\phi)=V(\phi)-\frac{c_{1}}{c_{3}} \ln \left(c_{2}+c_{3} V(\phi)\right) .
$$

In the case of small $\frac{c_{3}}{c_{2}}$ (physically meaning a small coupling of internal energy $e$ to potential energy $V$ ) this leads to

$$
\tilde{V}(\phi) \simeq-a V(\phi)+b V(\phi)^{2},
$$

where the constants $a$ and $b$ are functions of $c_{1}, c_{2}$ and $c_{3}$.

\section{Application to Scalar QED: Higgs Mechanism}

In principle, this scheme can be applied to all field theories given by an action $S[\phi]$. Equilibrium solutions are especially interesting for Scalar QED. Consider the Lagrangian

$$
\mathscr{L}=\frac{1}{4} F_{\mu v} F^{\mu v}+\left(D_{\mu} \phi\right)^{*}\left(D^{\mu} \phi\right)+V(|\phi|)
$$

with the potential

$$
V(|\phi|)=m^{2}|\phi|^{2}
$$

Let the fields undergo active Brownian motion, governed by equation (3.1) - the equilibrium limit in the small coupling expansion will give a QFT with the effective potential

$$
\tilde{V}(|\phi|)=-a V(|\phi|)+b V(|\phi|)^{2}=-a m^{2}|\phi|^{2}+b m^{4}|\phi|^{4},
$$

which is just the symmetry breaking potential of the Higgs mechanism. Conventionally expanding around one of the minima $|\phi|_{0_{ \pm}}= \pm \frac{1}{m}\left(\frac{a}{2 b}\right)^{\frac{1}{2}}$ leads to the identification of the gauge field mass and the Higgs mass:

$$
m_{A}^{2}=\frac{a}{b}\left(\frac{1}{m}\right)^{2} \quad, \quad m_{H}^{2}=2 a m^{2}
$$

\section{Summary and Outlook}

We sketched a procedure of how to deduce the Higgs mechanism within the Stochastic Quantization scheme. One could see the Higgs potential arising in a state, when active Brownian motion of the fields equilibrates. Further studies [8] involved the investigation also of nonequilibrium dynamics $\left(\frac{\partial e}{\partial s} \neq 0\right)$. So far, this was only done for finite degrees of freedom. Analogies of nonequilibrium behavior of active Brownian particles in QFT remain to be found. 


\section{References}

[1] C. W. Gardiner: Handbook of Stochastic Methods, Springer, Berlin 2004.

[2] G. Parisi, Y. Wu: Perturbation Theory without Gauge Fixing, Sci. Sin. 24, 483 (1981).

[3] P. Damgaard, H. Hüffel: Stochastic Quantization, Phys. Rep. 152, 227 (1987).

[4] M. Namiki: Stochastic Quantization, Springer, Berlin 1992.

[5] F. Schweitzer, W. Ebeling, and B. Tilch: Complex Motion of Brownian Particles with Energy Depots, Phys. Rev. Lett. 80, 5044 (1998).

[6] F. Schweitzer: Brownian Agents and Active Particles, Springer, Berlin 2003.

[7] A. Glück, H. Hüffel: Nonlinear Brownian Motion and Higgs Mechanism, Phys. Lett. B 659, 447 (2008).

[8] A. Glück, H. Hüffel, S. Ilijić: Canonical Active Brownian Motion, Phys. Rev. E 79, 021120 (2009). 